\title{
Design of A Magnet with Constant gradient field for Nuclear Magnetic Resonance
}

\author{
Lele Chen ${ }^{1,2, a}$, Changchun Zou ${ }^{1, b}$, Dianqing Sun ${ }^{2}$, Zhaobin $\mathrm{Gu}^{2}$ \\ ${ }^{1}$ China University of Geosciences (Beijing); \\ ${ }^{2}$ Research Institute of Petroleum Exploration \& Development-Langfang \\ aele_jobs@163.com, ${ }^{\text {b }}$ zoucc@cugb.edu.cn
}

Keywords: Nuclear Magnetic Resonance (NMR), Gradient Field, Magnet

\begin{abstract}
The principle of constant gradient field NMR is same with the logging expert system, the state-of-the -art technology in the world, wherein, gradient design and 2D spectrum inversion algorithm are adopted to quicken the instrument measurement speed while the diffusion coefficients of a fluid can be measured and the information obtained via the measurement of instrument can be more complete and authentic. The Article mainly introduces a magnet for gradient constant field NMR from such aspects as the parameter calculation of the gradient field, the selection of magnetic materials, finite element calculation and analysis, and detector installation and debugging. Experimental data shows that the design can completely realize fast NMR inspection and 2D spectrum inversion.
\end{abstract}

\section{Introduction}

Recently, there is a logging expert system introduced to the world, which adopts gradient fields, multi frequency, multi layer measurement and also 2D spectrum inversion calculation method. The system not only improves the measurement speed of the instrument but also extends the exploration depth further while obtaining more physical information of the rock, especially on the diffusion coefficients of molecules.

The gradient field NMR rock sample analysis instrument is an effective method for the laboratory study of 2D NMR spectroscopy. As to the said 2D NMR spectrum, one of the dimensions is transverse relaxation time T2 and the other is diffusion coefficient D. Both can be directly obtained from the 2D NMR spectrum. If compared with pulsed gradient technology, the rock sample analysis method for constant field NMR is same with the principle of the expert system and will set a foundation for the development of the state-of-the-art NMR instrument. Since the relaxation time and the diffusion coefficient of the liquid are mutually independent, 2D NMR spectrum can confirm the liquid type directly so as to separate oil, air and water fast and to provide a new approach to identify liquid types.

\section{Magnet with Gradient Field}

The creation of a constant gradient field is the core technology of the 2nd generation of NMR instrument. The field shall meet the requirements on both transverse evenness and longitudinal linearity. This section mainly explains such steps as the design, the calculation and the preparation of a magnet with a gradient constant field for NMR.

\subsection{Design of Magnet}

Currently, there is no precedent of designing such a magnet with a constant gradient field. But since the application of electromagnetic field calculation is very mature, the calculation method provides a theoretical basis for the calculation and analysis of constant gradient field. We can refer to the current calculation method of electro-magnetic field, and use finite difference method, finite element method, integral equation method and so on to design and calculate the constant gradient 
field. First, we can determine the dimensions of each part of the magnet and then optimize and correct each part of the magnet via 2D and 3D finite element analysis so as to reach the best result.

\section{2..1.1 Magnet Parameter Design}

Designed parameters of the constant gradient field are:

1. Field strength at the center $\quad B_{0}=700 \sim 1200 G s$

2. Field gradients $20 \mathrm{Gs} / \mathrm{cm}$

3. Linearity of field gradients $\leq \pm 5 \%$

\subsubsection{Selection of Magnetic Materials}

During the selection of magnetic materials, there are tree major aspects to consider, which are max energy product, intrinsic coercive force and $\mathrm{Br}$ temperature coefficient. The nature of the magnetic material is the most important factor among the said three aspects. Therefore, the selection of a suitable magnetic material is a key to the development of this magnet. The features of two magnetic materials are listed in below. See table 1.1.

Table 1 Features of Permanent Magnetic Materials

\begin{tabular}{|c|c|c|c|c|}
\hline Material & $\begin{array}{c}\text { Max Energy } \\
\text { Product } \\
\text { (Unit: KJ/m³) }\end{array}$ & $\begin{array}{c}\text { Intrinsic } \\
\text { Coercive Force } \\
\text { (Unit: KA/M) }\end{array}$ & $\begin{array}{c}\mathrm{Br} \\
\text { Temperature } \\
\text { Coefficient } \\
(\%)\end{array}$ & $\begin{array}{c}\text { Max } \\
\text { Operation } \\
\text { Temperature }\end{array}$ \\
\hline $\mathrm{NeFeB}(250 / 240)$ & 266 & 2560 & -0.12 & $80-200^{\circ} \mathrm{C}$ \\
\hline 2:17SmCo II (150/210) & $200-220$ & $1660-2100$ & -0.03 & $150-250^{\circ} \mathrm{C}$ \\
\hline
\end{tabular}

According to Table 1, 2:17SmCo has a better temperature reliability and will be the better choice in order to guarantee the reliability of the gradient field and the gradient linearity.

\subsubsection{Design of Magnet Shape and Slight Field Adjustment}

Purpose to Design the Shape of the Magnet.

The magnet shape decides the general distribution of the magnetic field. If magnetic flux leakage and calculation precision are taken into consideration, both 2D mode and 3D modes have a certain difference with the reality. Therefore, after the design of the overall shape of the magnet, slight adjustment is still required to the magnet so as to cover the design defect and to meet the requirements for use. See figure 1 for the shape of the magnet.

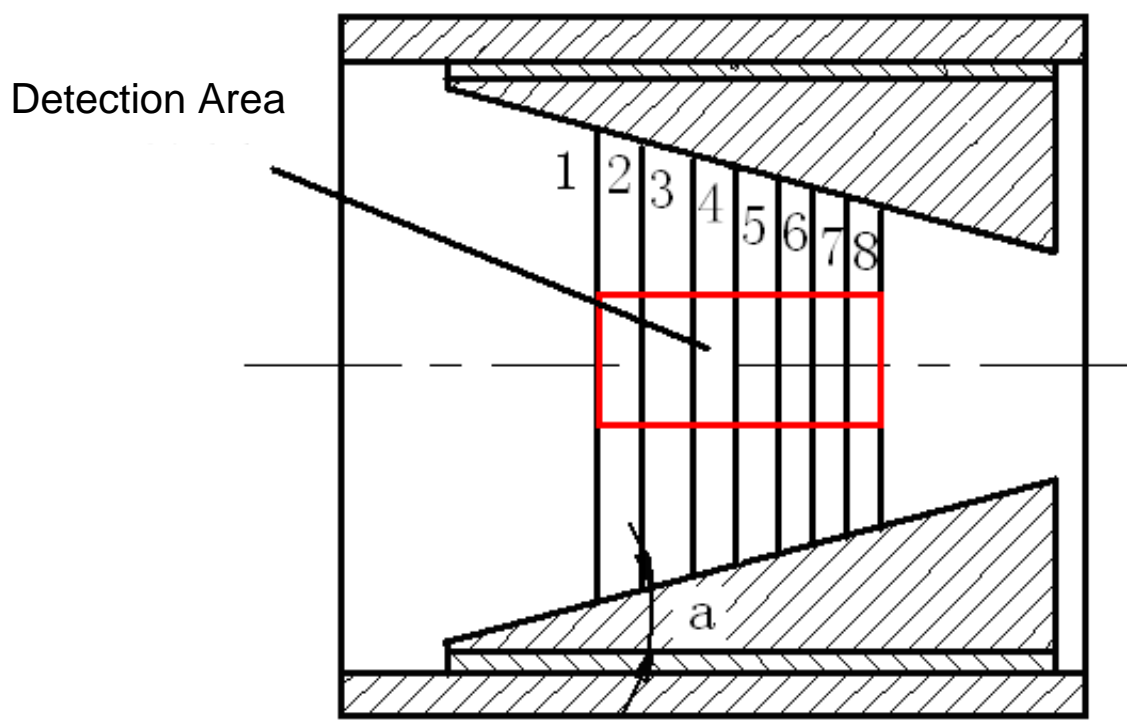

Figure 1 Distribution of Constant Gradient Field 
Figure 1 shows the distribution of the gradient field within the detection area. The magnetic flux density increases from No. 1 to No. 8 gradient and the field distribution at No.1-8 gradient can be detected via the probe of Gauss meter.

\subsection{Finite Element Calculation}

A static magnetic field can be expressed via below formulas:

$$
\begin{aligned}
& \nabla \cdot B=0 \\
& \nabla \times H=J
\end{aligned}
$$

$B=\mu H$

Magnetic flux density $\mathrm{B}$ can be expressed by

$$
B=\nabla \times A
$$

A second order differential equation as below can be obtained from formula (1-1)-(1-4):

$$
\nabla \times\left(\frac{1}{\mu} \nabla \times A\right)=J
$$

In the formula, $\mathrm{B}$ is the magnetic flux density, $\mathrm{H}$ magnetic induction, $\mathrm{J}$ current density, $\mu$ magnetic conductivity of the material and A vector potential. If $\nabla \cdot A=0$ is set, the unique value of $\mathrm{A}$ can be determined via finite element calculation of Formula (1) and the corresponding boundary conditions. And after post processing, magnetic flux density and the field quantity thereof can be derived.

\section{3D Finite Element Analysis}

\subsection{Creation of a Controlling Equation for the 3D Static Field and Set-up of Boundary} Conditions

3D static field meets below vector equation:

$$
\nabla \times\left(\frac{1}{\mu_{r}} \nabla \times A\right)=\mu_{0} J
$$

Dirichlet boundary condition is applicable there

$$
\hat{n} \times A=P
$$

And so is Neumann boundary condition for symmetric planes

$$
\hat{n} \times(\nabla \times A)=0
$$

Apply $\mu_{r}$ the continuous condition on abrupt interface

$$
\hat{n} \times A^{+}=\hat{n} \times A^{-}
$$

And

$$
\frac{1}{\mu_{r}^{+}} \hat{n} \times \nabla \times A^{+}=\frac{1}{\mu_{r}^{-}} \hat{n} \times \nabla \times A^{-}
$$

\subsection{Variational Formula}

According to variational principle, Formula (7)-(11) can be solved via the limit value of below functional under Formula (7) and (8).

$$
F(A)=\frac{1}{2} \iiint_{V} \frac{1}{\mu_{r}}(\nabla \times A) \bullet(\nabla \times A) d V-\mu_{0} \iiint_{V} J \bullet A d V
$$

\subsection{Division of Magnetic Area}

To identify each unit, a $4 \times M$ data array is introduced to code each unit and expressed with $n(i, e)$, wherein, $i=1,2,3$, and $e=1,2,3, \cdots, M \circ$. M presents the total number of units. 
Besides the above data, the finite element formula still requires other below data:

(1) $x_{i}, y_{i}, z_{i}(i=1,2,3, \cdots, N)$, Coordinate of each nodal point and $\mathrm{N}$ presents the total number of nodal points;

(2) Values of $\mu_{r}, \mu_{0}$ and $j_{z}$ of each unit;

((3) For corresponding value of $p$ of the nodal point on $\Gamma_{1}$, paralleled condition shall be reinforced to make $p=0$;

(4) $\Gamma_{2}$ Values of $\gamma$ and $q$ on each line segment.

\subsection{Element Interpolation}

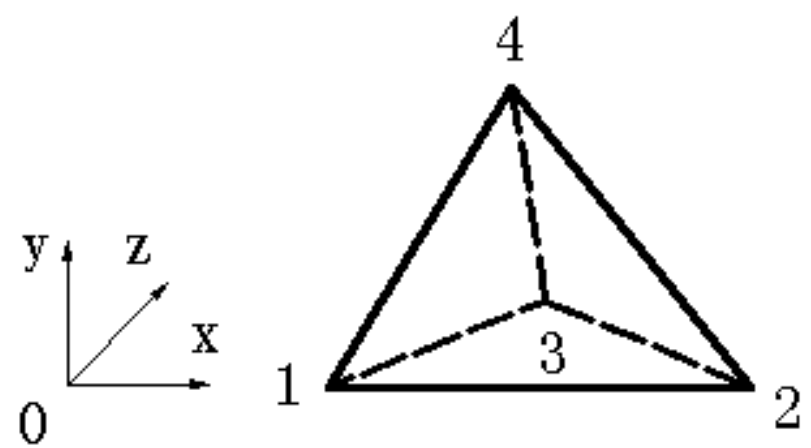

Figure 2 A Tetrahedron Unit of 3D Finite Elements

The unknown Function A within the tetrahedron unit is approximate to:

$$
A^{e}(x, y, z)=a^{e}+b^{e} x+c^{e} y+d^{e} z
$$

Formula (12) is introduced to the for nodal points to determine the four coefficients $a^{e} 、 b^{e} 、 c^{e}$ 和 $d^{e}$. If the value of $j$ on No. $A$ nodal point is marked as $A_{j}^{e}$, then below formulas can be obtained:

$$
\begin{aligned}
& A_{1}^{e}(x, y, z)=a^{e}+b^{e} x_{1}+c^{e} y_{1}+d^{e} z_{1} \\
& A_{2}^{e}(x, y, z)=a^{e}+b^{e} x_{2}+c^{e} y_{2}+d^{e} z_{2} \\
& A_{3}^{e}(x, y, z)=a^{e}+b^{e} x_{3}+c^{e} y_{3}+d^{e} z_{3} \\
& A_{4}^{e}(x, y, z)=a^{e}+b^{e} x_{4}+c^{e} y_{4}+d^{e} z_{4}
\end{aligned}
$$

According to the above formulas, below can be available:

$$
\begin{aligned}
a^{e} & =\frac{1}{6 V^{e}}\left|\begin{array}{llll}
A_{1}^{e} & A_{2}^{e} & A_{3}^{e} & A_{4}^{e} \\
x_{1}^{e} & x_{2}^{e} & x_{3}^{e} & x_{4}^{e} \\
y_{1}^{e} & y_{2}^{e} & y_{3}^{e} & y_{4}^{e} \\
z_{1}^{e} & z_{2}^{e} & z_{3}^{e} & z_{4}^{e}
\end{array}\right|=\frac{1}{6 V^{e}}\left(a_{1}^{e} A_{1}^{E}+a_{2}^{e} A_{2}^{E}+a_{3}^{e} A_{3}^{E}+a_{4}^{e} A_{4}^{E}\right) \\
b^{e} & =\frac{1}{6 V^{e}}\left|\begin{array}{cccc}
1 & 1 & 1 & 1 \\
A_{1}^{e} & A_{2}^{e} & A_{3}^{e} & A_{4}^{e} \\
y_{1}^{e} & y_{2}^{e} & y_{3}^{e} & y_{4}^{e} \\
z_{1}^{e} & z_{2}^{e} & z_{3}^{e} & z_{4}^{e}
\end{array}\right|=\frac{1}{6 V^{e}}\left(b_{1}^{e} A_{1}^{E}+b_{2}^{e} A_{2}^{E}+b_{3}^{e} A_{3}^{E}+b_{4}^{e} A_{4}^{E}\right)
\end{aligned}
$$




$$
\begin{aligned}
c^{e} & =\frac{1}{6 V^{e}}\left|\begin{array}{cccc}
1 & 1 & 1 & 1 \\
x_{1}^{e} & x_{2}^{e} & x_{3}^{e} & x_{4}^{e} \\
A_{1}^{e} & A_{2}^{e} & A_{3}^{e} & A_{3}^{e} \\
z_{1}^{e} & z_{2}^{e} & z_{3}^{e} & z_{4}^{e}
\end{array}\right|=\frac{1}{6 V^{e}}\left(c_{1}^{e} A_{1}^{E}+c_{2}^{e} A_{2}^{E}+c_{3}^{e} A_{3}^{E}+c_{4}^{e} A_{4}^{E}\right) \\
d^{e} & =\frac{1}{6 V^{e}}\left|\begin{array}{cccc}
1 & 1 & 1 & 1 \\
x_{1}^{e} & x_{2}^{e} & x_{3}^{e} & x_{4}^{e} \\
y_{1}^{e} & y_{2}^{e} & y_{3}^{e} & y_{4}^{e} \\
A_{1}^{e} & A_{2}^{e} & A_{3}^{e} & A_{4}^{e}
\end{array}\right|=\frac{1}{6 V^{e}}\left(d_{1}^{e} A_{1}^{E}+d_{2}^{e} A_{2}^{E}+d_{3}^{e} A_{3}^{E}+d_{4}^{e} A_{4}^{E}\right)
\end{aligned}
$$

Wherein,

$$
V^{e}=\frac{1}{6}\left|\begin{array}{cccc}
1 & 1 & 1 & 1 \\
x_{1}^{e} & x_{2}^{e} & x_{3}^{e} & x_{4}^{e} \\
y_{1}^{e} & y_{2}^{e} & y_{3}^{e} & y_{4}^{e} \\
z_{1}^{e} & z_{2}^{e} & z_{3}^{e} & z_{4}^{e}
\end{array}\right| \quad \text { Unit Cube }
$$

If the functional determinant is expended, then $a_{j}^{e} 、 b_{j}^{e} 、 c_{j}^{e}$ 和 $d_{j}^{e}$ can be determined.

And then if the formulas of $a_{j}^{e} 、 b_{j}^{e} 、 c_{j}^{e}$ 和 $d_{j}^{e}$ are introduced to Formula (12), below can be available:

$$
A^{e}(x, y, z)=\sum_{j=1}^{4} N_{j}^{e}(x, y, z) A_{j}^{e}
$$

Wherein, the interpolation function $N_{j}^{e}(x, y, z)$ is as below:

$$
N_{j}^{e}(x, y, z)=\frac{1}{6 V^{e}}\left(a_{j}^{e}+b_{j}^{e} x+c_{j}^{e} y+d_{j}^{e} z\right)
$$

Wherein, the interpolation function has below feature:

$$
N_{j}^{e}(x, y, z)=\delta_{i j}= \begin{cases}1 & i=j \\ 0 & i \neq j\end{cases}
$$

\subsection{Finite Element Calculation}

First, Formula (12) is expressed in scalar and component as below:

$$
\begin{gathered}
F(A)=\frac{1}{2} \iiint_{V} \frac{1}{\mu_{r}}\left[\left(\frac{\partial A_{Z}}{\partial Y}-\frac{\partial A_{Y}}{\partial Z}\right)^{2}+\left(\frac{\partial A_{X}}{\partial Z}-\frac{\partial A_{Z}}{\partial X}\right)^{2}+\left(\frac{\partial A_{Y}}{\partial X}-\frac{\partial A_{X}}{\partial Y}\right)^{2}\right. \\
\left.-\mu_{0}\left(A_{X} J_{X}+A_{Y} J_{Y}+A_{Z} J_{Z}\right)\right] d V
\end{gathered}
$$

Formula (16) is discreted and in Illustration 2, Component $A_{X} 、 A_{Y}$ 和 $A_{Z}$ are expressed as below:

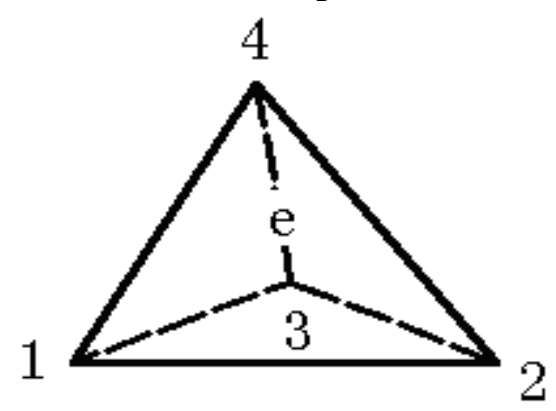

Figure 3 Tetrahedron Unit within Discrete Region 


$$
\begin{aligned}
& A_{x}^{e}=\sum_{j=1}^{4} N_{j}^{e} A_{z j}^{e}=\left\{N^{e}\right\}^{T}\left\{A_{x}^{e}\right\}=\left\{A_{x}^{e}\right\}^{T}\left\{N^{e}\right\} \\
& A_{y}^{e}=\sum_{j=1}^{4} N_{j}^{e} A_{y j}^{e}=\left\{N^{e}\right\}^{T}\left\{A_{y}^{e}\right\}=\left\{A_{y}^{e}\right\}^{T}\left\{N^{e}\right\} \\
& A_{z}^{e}=\sum_{j=1}^{4} N_{j}^{e} A_{z j}^{e}=\left\{N^{e}\right\}^{T}\left\{A_{z}^{e}\right\}=\left\{A_{z}^{e}\right\}^{T}\left\{N^{e}\right\}
\end{aligned}
$$

Formula (17) is inserted to Formula (16) to gain Part F of Unit e and then below can be obtained via the solution of the partial differential coefficients of $A_{x j}^{e} 、 A_{y j}^{e}, A_{z j}^{e}$.

$$
\begin{aligned}
& \left.\left.\left\{\frac{\partial F^{e}}{\partial A_{x}^{e}}\right\}=\left[K_{x x}^{e}\right]\right]\left\{A_{x}^{e}\right\}+\left[K_{x y}^{e}\right]\left\{A_{y}^{e}\right\}+\left[K_{x x}^{e}\right]\right]\left\{A_{z}^{e}\right\}-\left\{b_{x}^{e}\right\} \\
& \left\{\frac{\partial F^{e}}{\partial A_{y}^{e}}\right\}=\left[K_{y x}^{e}\right]\left\{A_{x}^{e}\right\}+\left[K_{y y}^{e}\right]\left\{A_{y}^{e}\right\}+\left[K_{y x}^{e}\right]\left\{A_{z}^{e}\right\}-\left\{b_{y}^{e}\right\} \\
& \left\{\frac{\partial F^{e}}{\partial A_{z}^{e}}\right\}=\left[K_{z x}^{e}\right]\left\{A_{x}^{e}\right\}+\left[K_{z y}^{e}\right]\left\{A_{y}^{e}\right\}+\left[K_{z z}^{e}\right]\left\{A_{z}^{e}\right\}-\left\{b_{z}^{e}\right\}
\end{aligned}
$$

All units are organized and the conditions of stagnation points are strengthened to get below simultaneous equations:

$$
\begin{aligned}
& \left\{\left\{\frac{\partial F}{\partial A_{x}}\right\}=\sum_{e=1}^{M}\left(\left[\overline{K_{x x}^{e}}\right]\left\{A_{x}^{e}\right\}+\left[\overline{K_{x y}^{e}}\right]\left\{A_{y}^{e}\right\}+\left[\overline{K_{x z}^{e}}\right]\left\{A_{z}^{e}\right\}-\left\{b_{x}^{e}\right\}\right)=\{0\}\right. \\
& \left\{\left\{\frac{\partial F}{\partial A_{x}}\right\}=\sum_{e=1}^{M}\left(\left[\overline{K_{y x}^{e}}\right]\left\{A_{x}^{e}\right\}+\left[\overline{K_{y y}^{e}}\right]\left\{A_{y}^{e}\right\}+\left[\overline{K_{y z}^{e}}\right]\left\{\overline{A_{z}^{e}}\right\}-\left\{b_{y}^{e}\right\}\right)=\{0\}\right. \\
& \left\{\frac{\partial F}{\partial A_{x}}\right\}=\sum_{e=1}^{M}\left(\left[\overline{K_{z x}^{e}}\right]\right]\left[\overline{A_{x}^{e}}\right\}+\left[\overline{K_{z y}^{e}}\right]\left\{\left(\overline{A_{y}^{e}}\right\}+\left[\overline{K_{z z}^{e}}\right]\left\{\left(\overline{A_{z}^{e}}\right\}-\left\{\hat{b_{z}^{e}}\right\}\right)=\{0\}\right.
\end{aligned}
$$

Formula (19) is re-organized as below:

$$
\left[\begin{array}{lll}
K_{x x} & K_{x y} & K_{x z} \\
K_{y x} & K_{y y} & K_{y z} \\
K_{z x} & K_{z y} & K_{z z}
\end{array}\right]\left\{\begin{array}{l}
A_{x} \\
A_{y} \\
A_{z}
\end{array}\right\}=\left\{\begin{array}{l}
b_{x} \\
b_{y} \\
b_{z}
\end{array}\right\}
$$

Dirichlet boundary condition is strengthened, namely the magnetic potential of each nodal point can be solved via the above formula.

According to the above principle, the electro-magnetic module of ANSYS, a finite element analysis software, is used to calculate magnetic vector $A_{z}$ and then magnetic flux density $B=\nabla \times\left(A_{Z} \hat{Z}\right)$ is obtained via $B_{0}$.

\section{Process and Assembly of the Magnet}

\subsection{Magnet Process}

(1) A polar plate is processed as Figure 4 shows. 

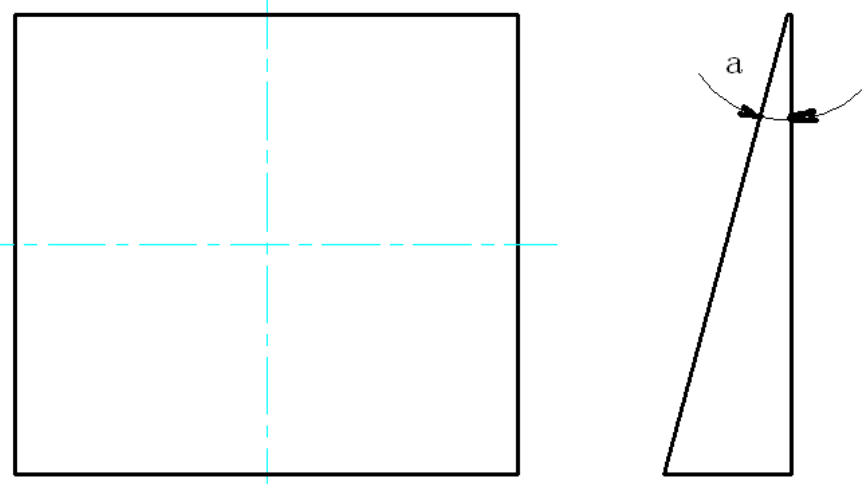

Figure 4 Polar Plate of the schematic

The polar plate is used to control the distribution of magnetic field within the gap via the shape of two corresponding polars, thus, Angle a must be guaranteed to a tolerance of \pm 0.005 during the process from the aspect of a constant gradient field.

(2) The process of magnetic yoke includes the process of a yoke plate and a side yoke plate. In order to guarantee the un-saturation of magnetic flux, it can do if the thickness of the magnetic yoke section is less than $25 \mathrm{~mm}$ as per calculation.
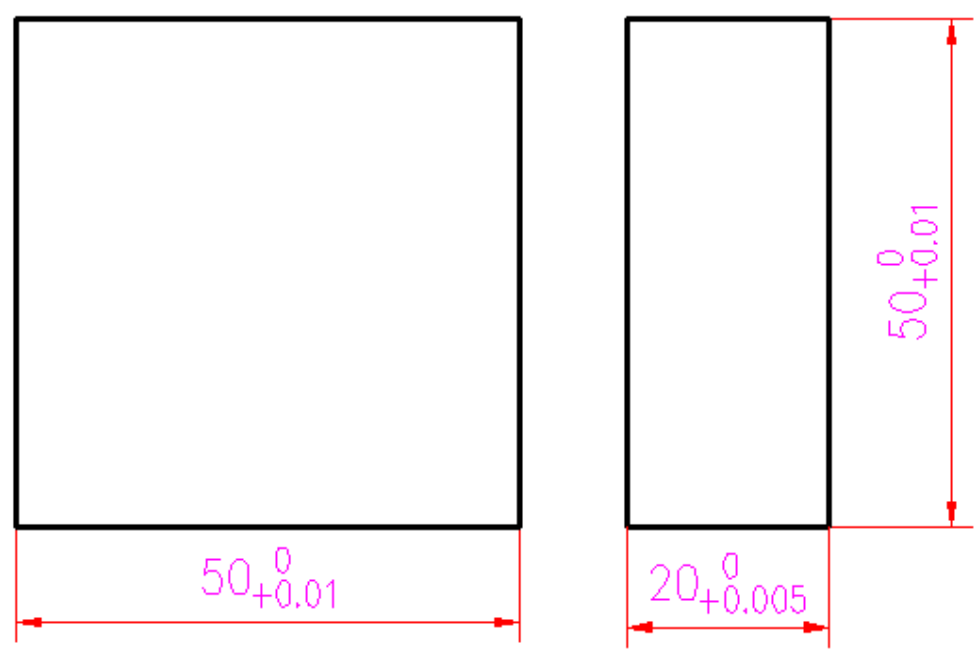

Figure 5 Magnetic Material

(3) Process of Magnetic Material

The magnet with a constant gradient field is made of 2:17SmCo the features of which decides that mechanical process is not suitable and that only linear cutting can do. To guarantee the assembly of the magnet, the detail dimensions shown in Figure 5 must be implemented.

\subsection{Magnet Assembly}

Since the magnetic material cannot be directly pasted on the yoke plate due to the existence of strong repelling force, magnetic blocks shall be first pasted on locating blocks which are ladder-shaped and locked to the yoke plate with locking screws. See Figure 6. 


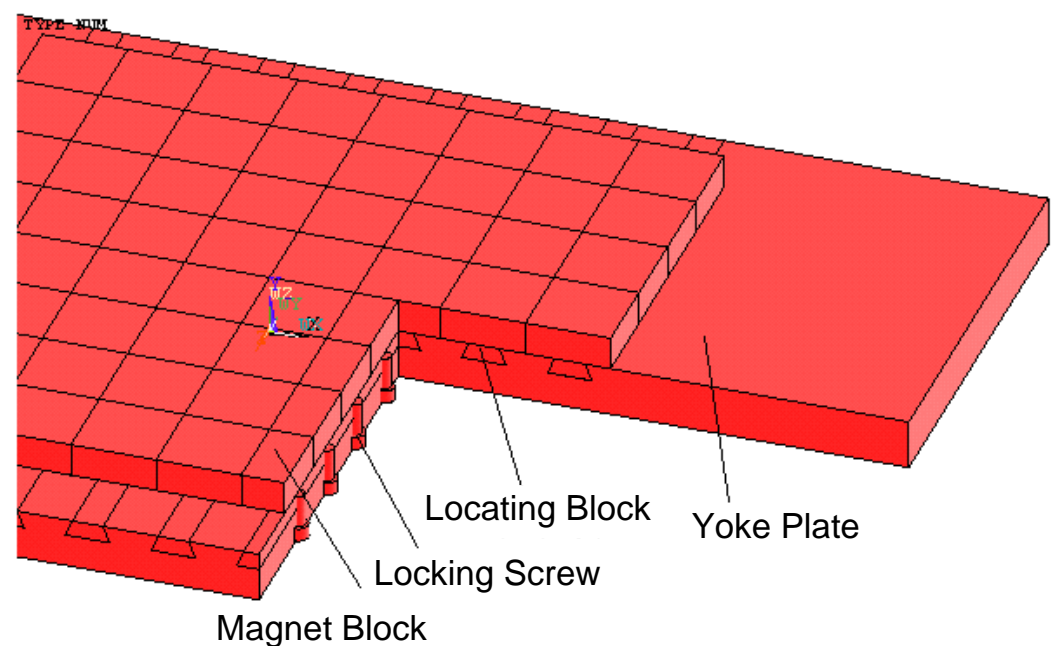

Figure 6 Paste of Magnet

The magnet with a constant gradient field is the most important part to this constant gradient field NMR rock sample analysis system which requires a magnetic field with a certain gradient force and gradient linearity. And the said magnetic field shall be stable to the situations when external temperature is not changed largely. The precise calculation, process, assembly and debugging to the magnet are required to get a constant gradient field.

\section{Experimental Results}

NMR tests are implemented to 6pcs of alkane samples separately and the relaxation time and diffusion coefficient of the alkane samples can be obtained from the test data via linear regression calculation.

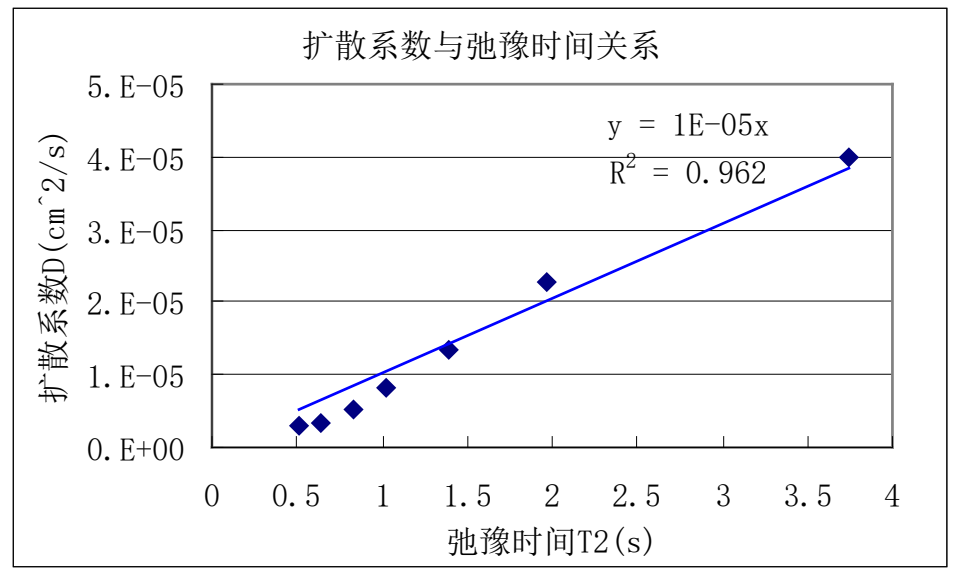

Figure 7 Relationship between Diffusion Coefficient D and Relaxation Time $T_{2 B}$ of Alkane

According to the illustration, the linearity values gained from the linear regression function are very high, all above 0.99 , and the relaxation time $T_{2 B}$ and diffusion coefficient $\mathrm{D}$ of alkane decrease as per the increase of carbon content. 


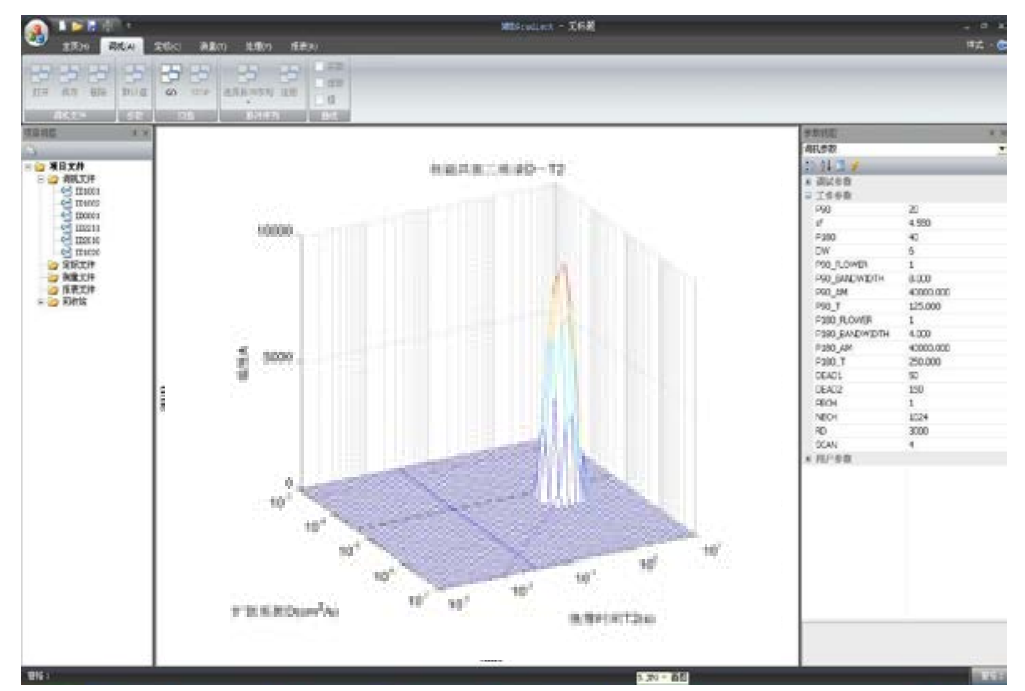

Figure 8 2D NMR Spectrum of Kerosene

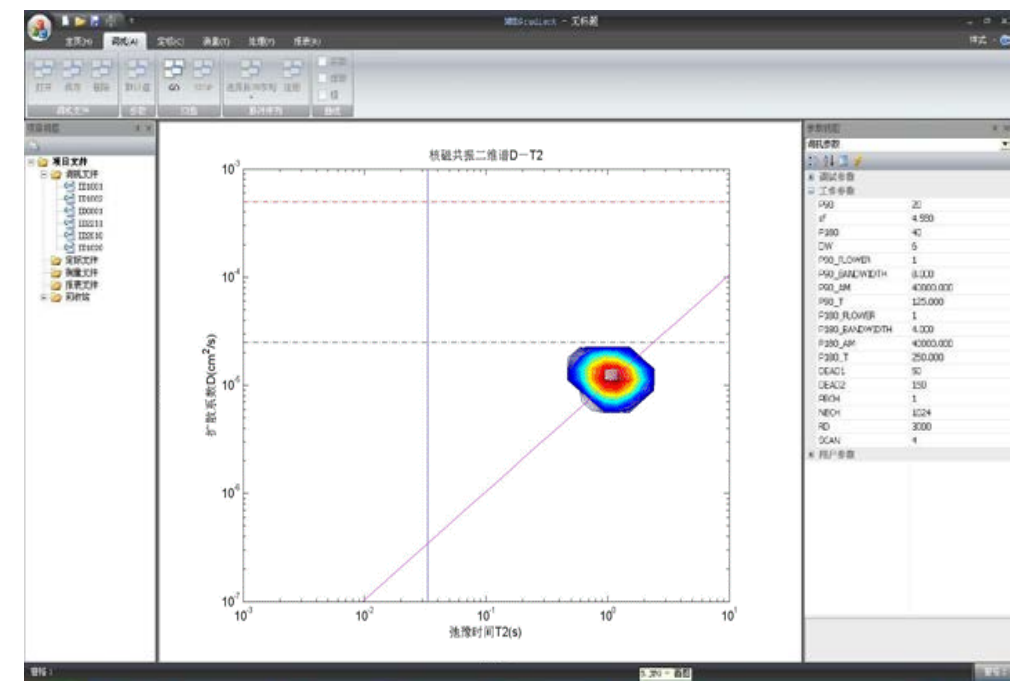

Figure 9 Contour Chart of the 2D NMR Spectrum of Kerosene

Figure 8 is the 2D NMR spectrum of kerosene and Figure 9 is the corresponding contour chart of the $2 \mathrm{D}$ spectrum. According to the figures, the 2D NMR spectrum of kerosene is on $D-T_{2}$ relation line, which shows that the diffusion coefficient and relaxation time of the raw oil have linear dependence relation.

\section{Conclusion}

1. The core technology of the NMR instrument is the design of magnet, which impacts the strength and precision of the NMR signal and the signal-noise ratio necessary to $2 \mathrm{D}$ spectrum inversion calculation.

2. As viewed from the experimental data, the magnet with constant gradient field for NMR can be realized from the 2D spectrum inversion and the accuracy and repeatability can reach industrial requirements.

3. In order to improve the signal-noise ratio of the NMR instrument, field strength, transverse evenness and longitudinal linearity of the field shall be adjusted properly. During numerical simulation, if such complex factors as magnetic flux leakage and temperature variation are taken into consideration, more precise design data can be obtained and the overall performance of the magnet can be improved. 


\section{References}

[1] D.Marschall et al. Method for Correlating NMR Relaxometry and Mercury Injection Data[J], SCA-9511, 1995.

[2] R.L.Kleinberg et al. Nuclear Magnetic Resonance of Rocks: $T_{1}$ vsT $_{2}[A]$. SPE 26470, 1993.

[3] Boqin Sun, Keh-Jim Dunn. Core analysis with two dimensional NMR[J]. SCA2002-38.

[4] Martin D, Charles Flaum. Nuclear Magnetic Resonance Method and Logging Apparatus for Fluid Analysis[P]. US:6891369, 2005-5-10.

[5] Timur A. Producible porosity and permeability of sandstone investigated through nuclear magnetic resonance principles[J]. Journal of Petroleum Technology, 1969,21,775 786.

[6] Brown, R. Protonrelaxation in crude oil[J],Nature, 1961,189.

[7] Seevers,D.O. A nuclear magnetic method for determining the permeability of sandstones[A]. Society of Professional Well Log Analysts, 1966.

[8] Partha P.Mitra, Pabitra N.Sen. Diffusion Propagator as a Probe of the Structure of Porous Media[J]. Physical Review Letters,1992,68(24):3555-3558.

[9] John P.Horkowitz et al. Residual Oil Saturation Measurements in Carbonates with Pulsed NMR Logs[A], The Log Analyst, March-April 1997.

[10] K.-J.Dunn, D.J.Bergman. Nuclear Magnetic Resonance Petrophysical and Logging Applications[M]. Pergamon: An Imprint of Elsebier Science,2002.

[11] M.B.Crowe et al. Measuring Residual Oil Saturation in West Texas Using NMR[A], SPWLA 38th Annual Logging Symposium, June 15-18,1997.

[12] S.Chen et al. Methods for Computing Swi and BVI from NMR Logs[A], SPWLA 39th Annual Logging Symposium, May 26-29,1998.

[13] Stefan Menger and Manfred Prammer. Can NMR Porosity Replace Conventional Porosity in Formation Evaluation[A]. SPWLA 39th Annual Logging Symposium, May 26-29, 1998.

[14] W.J.Looyestijn. Determination of Oil Saturation from Diffusion NMR Logs[A]. SPWLA37th Annual Logging Symposium, June 16-19,1996,S.

[15] G.R.Coates, H.J.Vinegar. Restrictive Diffusion From Uniform Gradient NMR Well Logging[J].SPE 26472,1993,575-590.

[16] Heinz W.Engl,Martin Hanker,Andreas Neubauer. Regularization of inverse problems[M]. Dordrecht, Kluwer academic publishers.1996 